26. Institute for Clinical and Economic Review. Modifications to the ICER value assessment framework for treatments for ultra-rare diseases. November 2017. Available at: https://icer-review.org/wp-content/ uploads/2017/11/ICER-Adaptations-of-Value-Framework-for-Rare-Diseases. pdf. Accessed December 4, 2018.

27. Bloomberg. FDA approves $\$ 450,000$-a-year treatment for rare disease. Fortune. August 11, 2018. Available at: http://fortune.com/2018/08/11/fdaalnylam-oppattro-approved/2018. Accessed December 4, 2018.
28. Pearson SD. The ICER value framework: integrating cost effectiveness and affordability in the assessment of health care value. Value Health. 2018;21(3):258-65.

29. Institute for Clinical and Economic Review. ICER value assessment framework. Available at: https://icer-review.org/methodology/icers-methods/ icer-value-assessment-framework/. Accessed December 4, 2018.

\title{
TTR Gene Silencers for Hereditary ATTR Amyloidosis: More than ICER Recognized
}

\author{
John L. Berk, MD
}

\section{COMMENTARY}

13, 2018, the Institute for Clinical and Economic Review (ICER), a nonprofit research organization created to provide impartial reviews and economic guidance to parties interested in new treatments approved by the U.S. Food and Drug Administration (FDA), presented its findings on 2 transthyretin (TTR) gene-silencing drugs to the Midwest Comparative Effectiveness Public Advisory Council (Midwest CEPAC), a committee of medical evidence experts. The products of billion-dollar investments by Alnylam Pharmaceuticals (Cambridge, MA) and Ionis Pharmaceuticals/Akcea Therapeutics (Carlsbad, CA) received grades of $\mathrm{B}+$ and $\mathrm{C}+$, respectively, on the scale of quality-adjusted life-years. For patients with hereditary transthyretin amyloidosis (hATTR) and their advocacy organizations committed to getting drugs to affected people, the lukewarm grading was surprising and disappointing.

Two months earlier, the New England Journal of Medicine featured 2 lead articles and an editorial, ${ }^{1-3}$ trumpeting diseasealtering randomized controlled trial (RCT) results for these TTR gene-silencing drugs-patisiran, an RNA interference agent, and inotersen, an antisense oligonucleotide drug-in the treatment of hATTR. hATTR is an autosomal dominant lethal neurodegenerative disease resulting from the misaggregation and organ deposition of mutated transthyretin. Advancing 10 times as quickly as diabetic polyneuropathy, patients with untreated hATTR amyloid polyneuropathy die 5-15 years after diagnosis from progressive loss of limb function, bowel control, and blood pressure regulation that renders them bed bound and completely dependent on others. It is a disease that devastates the afflicted and traumatizes family caregivers.

\section{J Manag Care Spec Pharm. 2019;25(1):15-16}

Copyright $\odot 2019$, Academy of Managed Care Pharmacy. All rights reserved.
The positive results of the patisiran and inotersen studies were preceded 5 years earlier by 2 RCTs that demonstrated the ability of TTR protein stabilizers to inhibit TTR misfolding and slow neurologic decline in patients with hATTR amyloidosis. ${ }^{4,5}$ Those drugs, diflunisal (generic nonsteroidal anti-inflammatory drug) and tafamidis (FoldRx/Pfizer), changed the lives of many patients. Despite an overall beneficial effect, however, over $75 \%$ of patients treated with TTR protein stabilizers experience continued neurologic decline. In contrast, the TTR gene silencers more reliably halt disease and, for the first time, offer potential improvement in polyneuropathy and, possibly, heart function in $37 \%-56 \%$ of those receiving treatment. ${ }^{1,2}$ Moreover, an ad hoc analysis report showed a decrease in all-cause mortality and cardiac hospitalization rates in patients randomized to the TTR gene silencer patisiran versus placebo patients. ${ }^{6}$

Why then the disparate findings and the lukewarm ICER/ Midwest CEPAC grading of TTR gene silencers?

To the external observer, the ICER time line from data review to drug grading appeared overly ambitious. Six months before the September 2018 Midwest CEPAC presentations, ICER posted scoping documents for the 2 topics on the meeting agenda: (a) use of antiandrogen therapies in nonmetastatic prostate cancer and (b) effectiveness of TTR gene silencers in hATTR amyloid neuropathy. However, full access to RCT data critical to ICER's proposed analyses and presentations did not occur until the data embargo imposed by the New England Journal of Medicine was lifted with the publication of study results for the antiandrogen products on April 12, 2018, and the publication of trial data for the TTR gene silencers on July 5 , 2018, nearly 3 months later. ${ }^{7,8}$ Despite the relative inaccessibility of the TTR gene silencing trial data, evidence reports for antiandrogen and TTR gene-silencer presentations were posted 
the last week of August 2018, a full 18 weeks after the release of antiandrogen RCT data but less than 8 weeks after TTR genesilencing RCT data became available.

To compound the challenges of a tight time line for analysis of the TTR gene-silencing RCT data, the patisiran and inotersen studies used a complex quantitative neurologic metric (modified neurologic impairment score +7 nerve attributes $[\mathrm{mNIS}+7])$ as the primary outcome measure of drug effect. To ICER's dismay, mNIS +7 did not easily correlate to the performance of activities of daily living.

Unable to understand the magnitude of neurologic progression in the placebo cohort captured by the mNIS+7 and quality of life metrics and, consequently, the clinical impact of patisiran and inotersen, ICER resorted to substituting insensitive secondary neurologic outcome measures for mNIS +7 scoring. These 2 secondary neurologic staging systems-the polyneuropathy disability (PND) score and familial amyloidotic polyneuropathy (FAP) staging-loosely correlate mNIS +7 with a patient's ability to walk unassisted, aided with canes, or when requiring wheelchair transport. ${ }^{9}$ Unfortunately, each PND or FAP stage encompasses a wide range of mNIS+7 scores, rendering these secondary neurologic scales too insensitive to accurately track disease progression in clinical trials.

In effect, deviating from the primary outcome measure $(\mathrm{mNIS}+7)$ in favor of a more understandable metric effectively minimized the detection of neurologic decline in the placebo arm as well as the neurologic protection afforded by TTR gene-silencing agents during the RCTs and proved fatal to the otherwise elegant economic modeling analyses performed by ICER. In short, if FDA approval of TTR gene silencers was not predicated on secondary neurologic measures, economic modeling should not be either.

The ICER analysis also raised questions about the clinical importance of several baseline demographic inequities between treatment arms in the patisiran and inotersen studies. On closer inspection of the data, longitudinal analyses and forest plot presentations minimized the import of these issues. Specifically, the TTR gene-silencing drug effect proved to be independent of V30M or V122I ATTR genotype, geographic representation (United States vs. other countries), New York Heart Association staging, and disparate study discontinuations from treatment and placebo arms. To ICER's credit, its analysis touched on an imbalance of cardiac deaths in partisiran-treated subjects. Subsequent publication by a blinded, independent clinical endpoint adjudication committee indicated low rates of cardiac deaths in patients with features of amyloid cardiomyopathy randomized to the placebo $(2.7 \%)$ and patisiran (5.6\%) treatment arms. ${ }^{6}$

In sum, the economic modeling and resulting qualityadjusted life-year cost assignments were undermined by an inability to translate the improvements in primary neurologic outcomes observed during TTR gene silencing to an understandable effect on patient lives, resulting in low economic grading of drug effect. ICER's earlier presentation of antiandrogen therapy effect on nonmetastatic prostate cancer survival — extending overall survival by 1.7 years—generated an A + rating by the Midwest CEPAC body of medicine evidence experts. Because neurologic deterioration drives mortality in patients with hATTR amyloid polyneuropathy, experts in the field anticipate that neurologic stabilization, or even improved heart and nerve function due to TTR gene-silencing treatments, will translate to survival benefits far exceeding those recorded for early antiandrogren treatment of patients with prostate cancer. By all clinical measures, TTR gene silencers appear to be here to stay.

\section{Author}

JOHN L. BERK, MD, Assistant Director, Amyloidosis Center, Boston University Medical Center, Boston, Massachusetts.

AUTHOR CORRESPONDENCE: John L.Berk, MD, 72 E. Concord St., K504, Boston, MA 02118. Tel.: 617-358-4750;

E-mail: jberk@bu.edu.

\section{DISCLOSURES}

No funding support was received for the writing of this commentary. The author serves as a consultant for Akcea Therapeutics, Alnylam Pharmaceuticals, Corino Therapeutics, Intellia Therapeutics, and Ionis Pharmaceuticals.

\section{REFERENCES}

1. Adams D, Gonzalez-Duarte A, O'Riordan WD, et al. Patisiran, an RNAi therapeutic, for hereditary transthyretin amyloidosis. N Engl J Med. 2018;379(1):11-21

2. Benson MD, Waddington-Cruz M, Berk JL, et al. Inotersen treatment for patients with hereditary transthyretin amyloidosis. N Engl J Med. 2018;379(1):22-31.

3. Buxbaum JN. Oligonucleotide drugs for transthyretin amyloidosis. N Engl J Med. 2018;379(1):82-85.

4. Berk JL, Suhr OB, Obici L, et al. Repurposing diflunisal for familial amyloid polyneuropathy: a randomized clinical trial. JAMA. 2013;310(24):2658-67.

5. Coelho T, Maia LF, Martins da Silva A, et al. Tafamidis for transthyretin familial amyloid polyneuropathy: a randomized controlled trial. Neurology. 2012;79(8):785-92.

6. Solomon SD, Adams D, Kristen A, et al. Effects of patisiran, an RNA interference therapeutic, on cardiac parameters in patients with hereditary transthyretin-mediated amyloidosis: an analysis of the APOLLO Study. Circulation. September 14, 2018 [Epub ahead of print]. Available at: https:// www.ahajournals.org/doi/10.1161/CIRCULATIONAHA.118.035831. Accessed November 27, 2018.

7. Smith MR, Saad F, Chowdhury S, et al. Apalutamide treatment and metastasis-free survival in prostate cancer. N Engl J Med. 2018;378(15):1408-18.

8. Hussain M, Fizazi K, Saad F, et al. Enzalutamide in men with nonmetastatic, castration-resistant prostate cancer. N Engl J Med. 2018;378(26):2465-74.

9. Adams D, Suhr OB, Dyck PJ, et al. Trial design and rationale for APOLLO, a phase III, placebo-controlled study of patirisan in patients with hereditary ATTR amyloidosis with polyneuropathy. BMC Neurology. 2017;17(1):181-93. 